\title{
IQOS: A Cross Layer Design Approach for Improve Quality of Service in Wireless Sensor Networks
}

\author{
Rakesh Kumar Saini ${ }^{1}$ and Ritika ${ }^{2}$ \\ ${ }^{1,2}$ Department of Computer Application, DIT University, Dehradun \\ Uttrakhand, India \\ ${ }^{1}$ rakeshcool2008@gmail.com, ${ }^{2}$ hod.mca@dituniversity.edu.in
}

\begin{abstract}
In Wireless Sensor Network, Sensors nodes have limited power and resources. Sensor nodes are used for monitoring particular area in wireless sensor network. Due to lack of limited resources, a sensor node does not work properly with traditional layered routing protocol. Traditional layered routing protocol allows interaction between wireless protocol stack layers adjacently but does not allow interaction between wireless protocol stack layers non-adjacently. Cross layer design approach can allow interaction between wireless protocol stack layers non-adjacently. Cross layer design approach allows sharing variables between layers. Developing cross layer design approach is most important for improve Quality of Service (QoS) parameters like Energy-efficiency, throughput and Average end to end delay in Wireless Sensor Networks. Thus our focus is to improve the Quality of Service $(Q o S)$ for Wireless Sensor Network. One of the ways to improve Quality of Service $(Q o S)$ is cross layer design approach. In this paper we propose a cross layer design approach (IQOS) which optimizes Quality of Service (QoS) factors of the sensor nodes at the Network Layer, Data Link Layer and Physical Layers of the wireless protocol stack because Quality of Service $(Q o S)$ factors exist in these three layers.
\end{abstract}

Keywords: Cross-layer, WSN Protocol stack, Sensor nodes, quality of service (QoS) support, Wireless Sensor Network

\section{Introduction}

Wireless sensor network is a network that is used to monitor the physical and environmental such as temperature, vibration, sound and so on. In traditional communication networks, the Open Systems Interconnection (OSI) layered architecture has been widely adopted and has served many communications systems well in the past; however, evolving wireless networks of today are seriously challenging this design philosophy. The layered architecture defines a stack of protocol layers in which each layer operate within its well-defined function and boundary, and thus allowing changes to the underlying technology at each layer without imposing the need to change the overall system architecture. Traditional layered approach has been successful in its ability to provide modularity, transparency and standardization in the wire line networks but might be unsuitable in the wireless sensor networks domain. In the recent years, many research works have been presented for wireless sensor networks which are based on the interaction between various non-adjacent layers of the wireless sensor network stack. Cross layer design is a co-operation between multiple layers that combine the resources and create a network that is highly adaptive. The cross layer design approach can increase energy efficiency of sensor nodes in wireless sensor network. The Cross-layered design approach in wireless sensor network is more useful, energy efficient, scalable and secure than with traditional approaches. Parameters which can be optimized by Cross layered design approach are throughput, network lifetime, quality-of-Service, resource constraint, 
scalability, functionality. The traditional layered approach follows strict layering principles and provides a platform for designing interoperable systems, but it suffers from more transfer overhead. So Cross-layered approach is used to minimize this overhead by having data and information shared among different layers. So there is requirement of a cross layer design approach that will improve quality-of-service for wireless sensors network. Cross layer design approach can allow interaction between layers. By using cross layer design approach, one layer can exchange data between different layers.

\section{Cross Layer Design}

Cross layer design is a approach that provides facility of exchanging information between layers. Cross. layer design allow interaction between different layers nonadjacently. Cross layer design approach is the best approach for improve Quality-ofService(QoS) in WSN. In Traditional layered protocol architecture one layer can exchange data and communicate between each other one after other. A protocol encapsulates data arrived from its upper adjacent layer and forwards it down to its lower adjacent layer. Similarly, it can decapsulate data received from its lower adjacent layer and forward it up to its upper adjacent layer. There is no interaction between any nonadjacent layers as in such a layered architecture. Traditional layered protocol architecture is suitable only for wired networks but it is not suitable for wireless networks. To overcome the drawbacks of traditional layered protocol architecture design in wireless sensor networks, researchers have proposed the cross-layer design approach. Cross layer design may be defined as breaking of OSI layer in communication network, Cross layer design allows not only protocol interactions across the layers, but also the possibilities of merging and removal of layers, and the creation of new interfaces and entities for interactions between the layers [19]. The traditional layered approach provides a platform for designing interoperable systems, but it suffers from more transfer overhead. So, Cross layered approach is used to minimize this overhead by having data and information shared among different layers. In this paper we have proposed a cross layer design approach (IQOS). Proposed IQOS will be more energy-efficient as compare to existing approach.

\section{Existing Cross Layer Design Approach}

A few cross-layer design approaches have been proposed for Wireless Sensor Network. There is a considerable amount of surveys in the literature that discuss WSN technologies in general [1]-[7].The complete literature survey have discuss in [8]-[16]. Irfan AI-Anbagi et al., [8] have proposed a cross layer scheme for quality of service in wireless sensor network for obtaining delay and reliability. This cross layer scheme provide better reliability. Qingwen Liu et al., [9] have proposed a scheduling mechanism for multiple connections for the increasing quality of service demand of different-different applications. This scheduling mechanism handles time diversity and frequency diversity. Alper K. Demir et al., [10] have proposed cross-layer architecture for quality of service. This proposed architecture is very useful for achieving reliability and others quality of service parameters. Ghalib A. Shah et al., [11] have describe a cross-layer approach for improvement quality of service by optimizing the functionalities of communication protocols. Rajeev Ranjan et al., [12] have proposed a cross-layer design approach for Wireless Sensor Network. This cross-layer design approach improves energy-efficiency of sensor nodes in wireless sensor network. Veronica Sentongo et al., [13] have proposed a cross-layer approach for improving end-user delay and per-user throughput of voice over internet protocol when packets size are increasing. Rafael Laufer et al., [14] have proposed Cross-Layer backpressure architecture for handling multihop networks by using TDMA MAC Protocol. David Espes et al., [15] have proposed a cross-layer routing protocol (PLOSA) to offer a high delivery rate, a low end-to-end delay \& a low energy consumption. PLOSA optimize sleeping periods of devices because each node can receive 
packets to be forwarded only in a specific part of the frame. Ramin Babaee et al., [16] have proposed an efficient routing algorithm with the goal of minimization of the end-toend outage probability from source to destination for Multihop Wireless Network.

\section{Proposed Cross Layer Design Approach(IQOS)}

Proposed cross-layer design approach (IQOS) allows interaction between layers. In IQOS, each layer interact with other layers and each layer has knowledge about others layers. We design IQOS as in Figure7 Firstly at Physical layer we calculate RSSI (Received Signal Strength indicator) Value of Sensor nodes from Base Station and send these RSSI values to Network layer. Secondly at Network Layer we check RSSI value of sensor nodes and if node has larger RSSI value then select this node as data forwarding node from sensor nodes to base station, Thirdly at MAC Layer we focused on avoiding collision and overhearing for this purpose a Scheduling algorithm is used here. Scheduling algorithm makes schedule of all sensor nodes that are coming from Network layer and provide error free transmission. Scheduling algorithm also increase flexibility. Scheduling algorithm provide high-quality data service, a multilevel of sensing coverage and network connectivity.

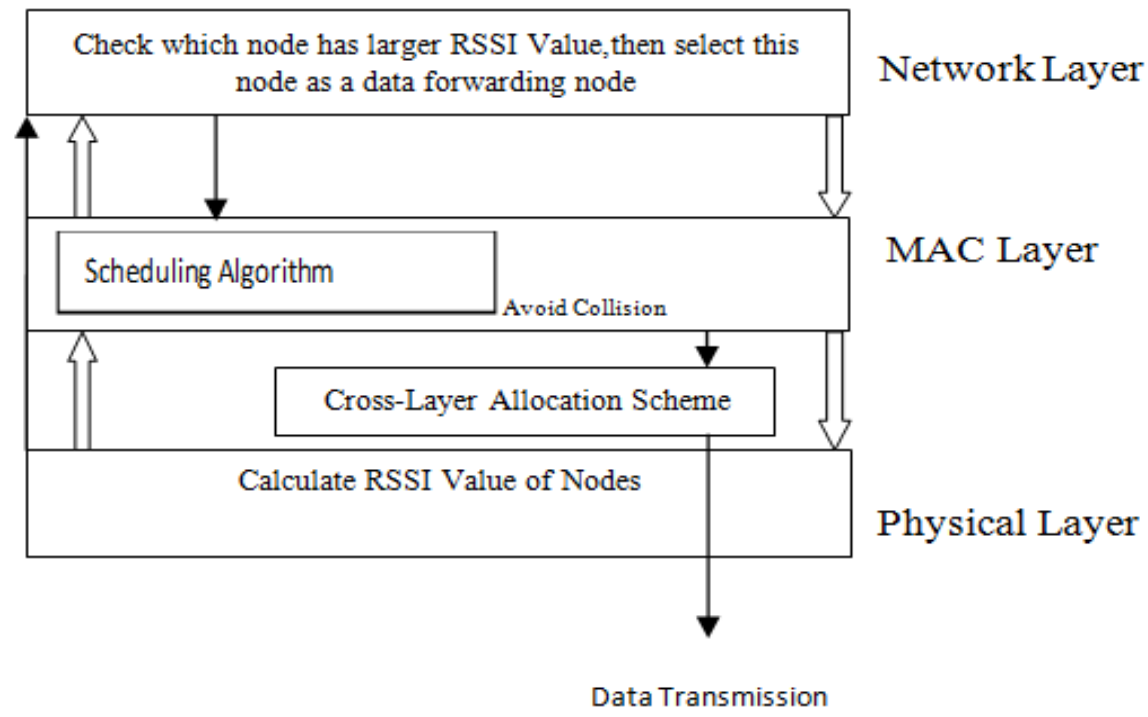

Figure 1. Proposed IQOS

Scheduling algorithm at MAC Layer is shown in algorithm 1. Scheduling algorithm make schedule of all sensor nodes that are coming from upper layer (Network layer).It check the channel whether it is free or not, if channel is free then give a time slot to sensor and dispatch packet to Cross layer allocation buffer (CLAB).CLAB check frequency, Channel and time slot of sensor nodes and if channel is free then dispatch packets from CLAB to Physical Layer.

Notations used:

$\mathrm{B}_{\mathrm{D}}$ - Buffer data

$\mathrm{P}_{\mathrm{N}}$ - Packets

CLAB - Cross layer allocation buffer

$\mathrm{T}_{\mathrm{s}}$-Time Slot

Ch - Channel 


\section{Algorithm 1: Scheduling Algorithm at MAC Layer}

1. If buffer data $\mathrm{B}_{\mathrm{D}}=\mathrm{P}_{\mathrm{N}}$ Then

2. Check Channel $\mathrm{Ch}$ whether it is free or not

3. If $\mathrm{Ch}=0$ then set $\mathrm{Ch}=\mathrm{P}_{\mathrm{N}}$

4. Set $\mathrm{P}_{\mathrm{N}}=\mathrm{T}_{\mathrm{s}}$

5. $\mathrm{P}_{\mathrm{N}}$ dispatch to CLAB buffer

6. CLAB Check $\mathrm{Ch}$ is free or not

7. If $\mathrm{Ch}=0$ then

8. Dispatch $\mathrm{P}_{\mathrm{N}}$ from CLAB

9. Repeat Step 1 to 8

\section{Performance Evaluation}

We develop a simulation environment to evaluate the efficiency of IQOS. For this purpose we are using QualNet 5.0.2 simulation modeling tool. We are using some QoS parameters like Average jitter, Throughput, Average end-to-end delay.

\section{Average Jitter}

Data from source to destination will reach the destination with different delays. A packet's delay varies with its position in the queues of the routers along the path between source and destination and this position can vary unpredictably. This variation in delay is known as Jitter.

\section{Throughput}

Throughput means total number of packets received by the Base Station. Throughput is the average rate of successful message delivery over communication channel.

\section{Average End-to-End Delay}

Average end-to-end delay is time in which data send from sensor node to the base station. Due to queuing and different routing paths, a data packet may take a longer time to reach its destination. The end-to-end delay experienced by the packets for each flow the individual packet delay is summed and the average is computed.

Table 1. Simulation Parameters

\begin{tabular}{|l|l|}
\hline \multicolumn{1}{|c|}{ Parameter } & \multicolumn{1}{c|}{ Value } \\
\hline Sensor nodes & $1,2,3,4,5,6,7,8,9,10$ \\
\hline Destination node & 11 \\
\hline Buffer Size & 1024 \\
\hline Terrain Range & $100 \mathrm{~m} \times 100 \mathrm{~m}$ \\
\hline No. of nodes & 10 \\
\hline Frequencies & $2.4 \mathrm{GHz}$ \\
\hline Traffic Type & CBR \\
\hline Channel Type & Wireless channel \\
\hline Protocols & AODV \\
\hline
\end{tabular}


The performance of proposed IQOS is verified in the experiment, the sensor nodes in Wireless Sensor Network are distributed randomly in the $100 \mathrm{~m} * 100 \mathrm{~m}$ area. In this simulation environment (Figure 2) sensor nodes 1,2,3,4,5,6,7,8,9,10 are co-operately pass their data to the destination node (Base Station) running simulation is shown in Figure 3. In Running simulation sensor nodes are sending packets to destination node 11(Base Station).

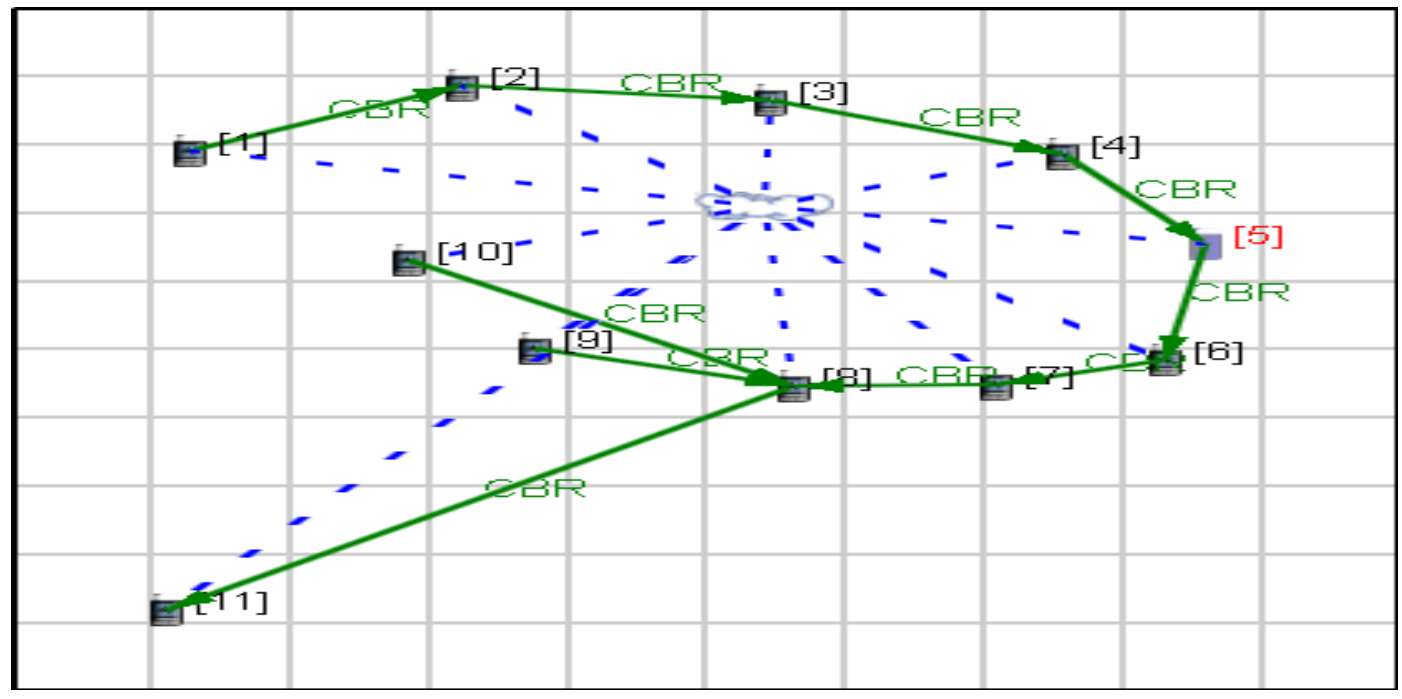

Figure 2. Simulation Environment

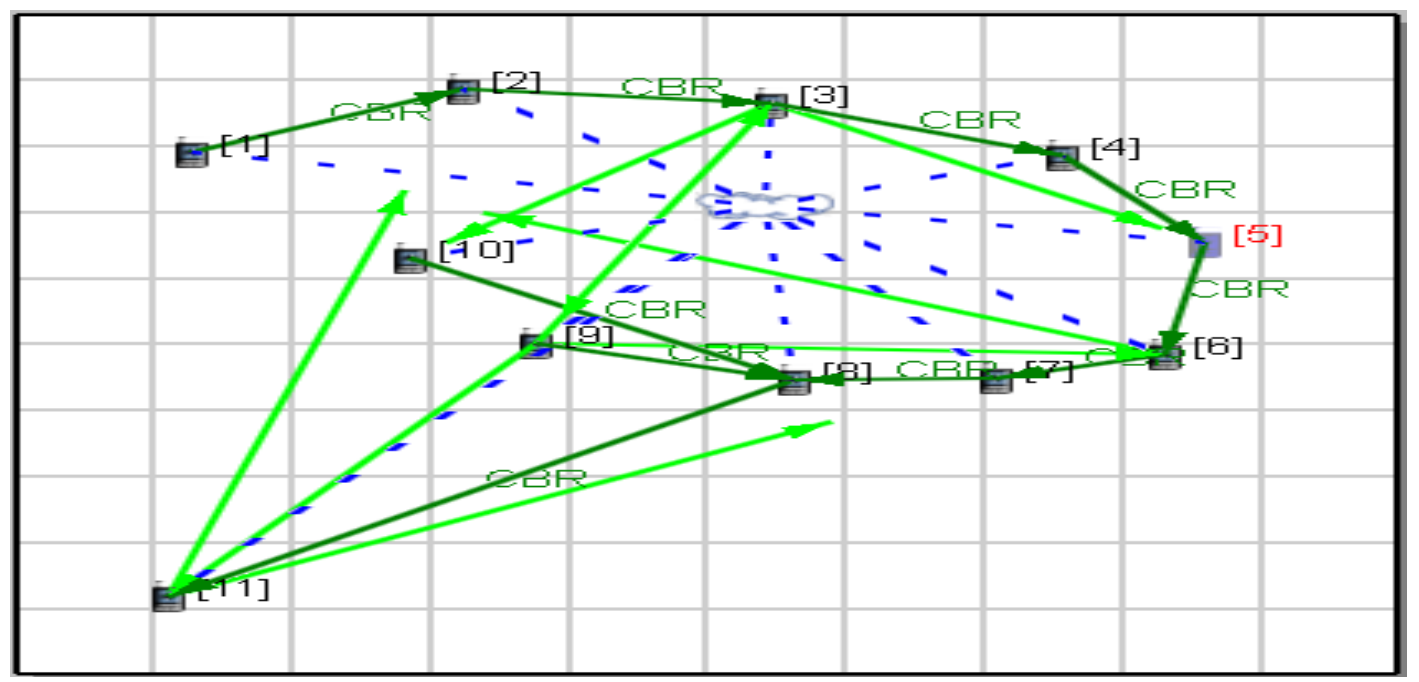

Figure 3. Running Simulation

\section{Simulation Results}

QualNet 5.0.2 simulator is used to implement the Proposed cross layer design approach. The Performance of Proposed cross layer design approach is evaluated. Table 1 lists the parameters used in the simulation. The key performance measures are Average jitter, Throughput and Average end-to-end delay. In Figure 4 show result Average jitter. Sensing data that are coming from sensor nodes to base station, Average jitter show the delay of sensing data from sensor nodes to base station. 


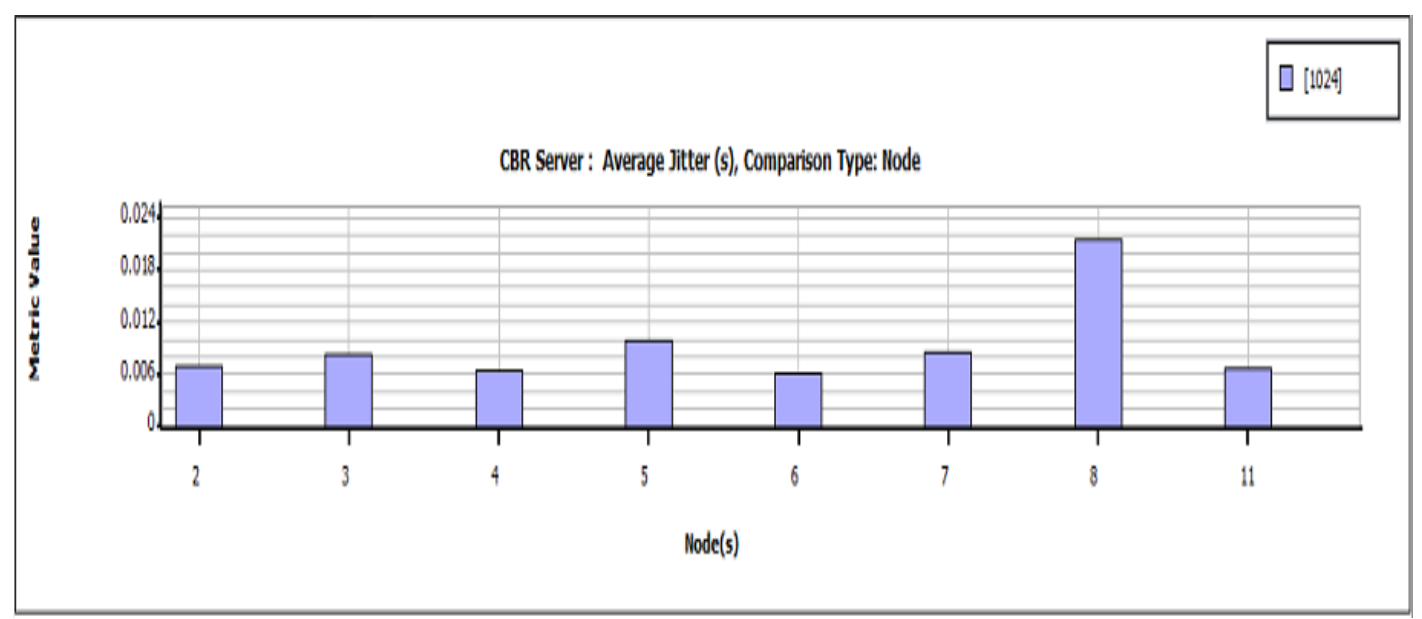

Figure 4. Average Jitter

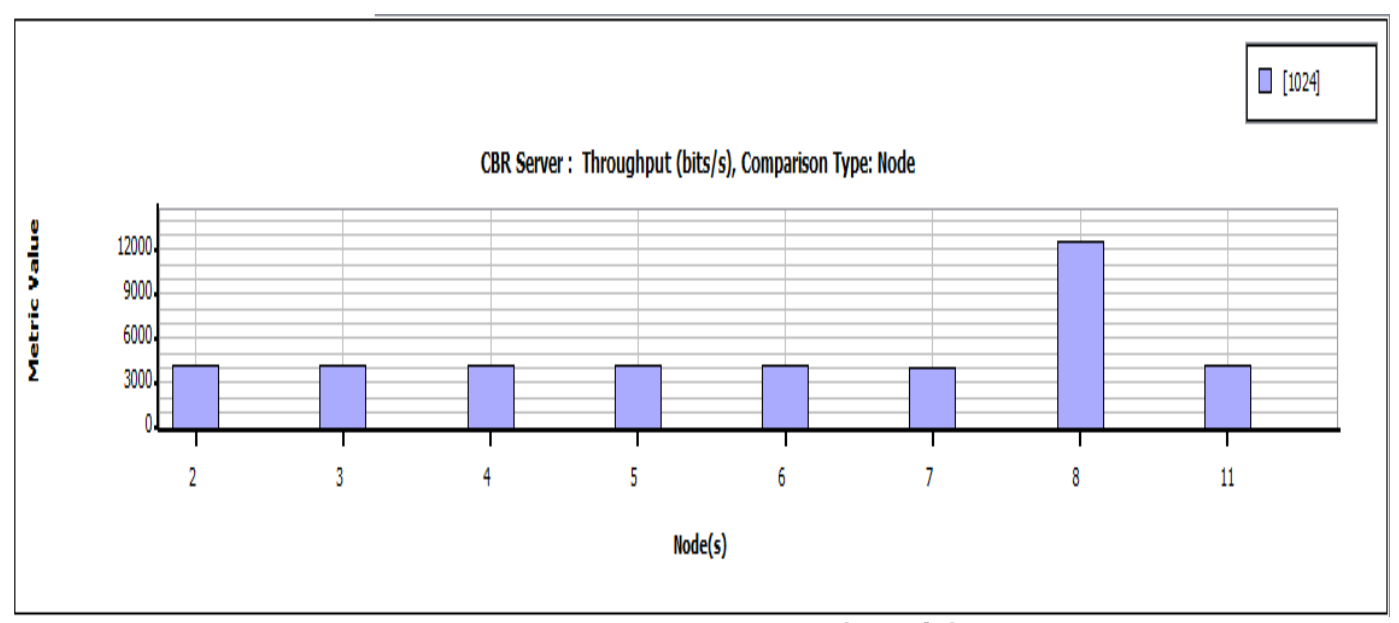

Figure 5. Throughput (bits/s)

In Figure 5 shown total number of packets received by base station. Sensor nodes 1, $2,3,4,5,6,7,8,9,10$ are sending packets to base station, there are base station received different-different packets from sensor nodes.

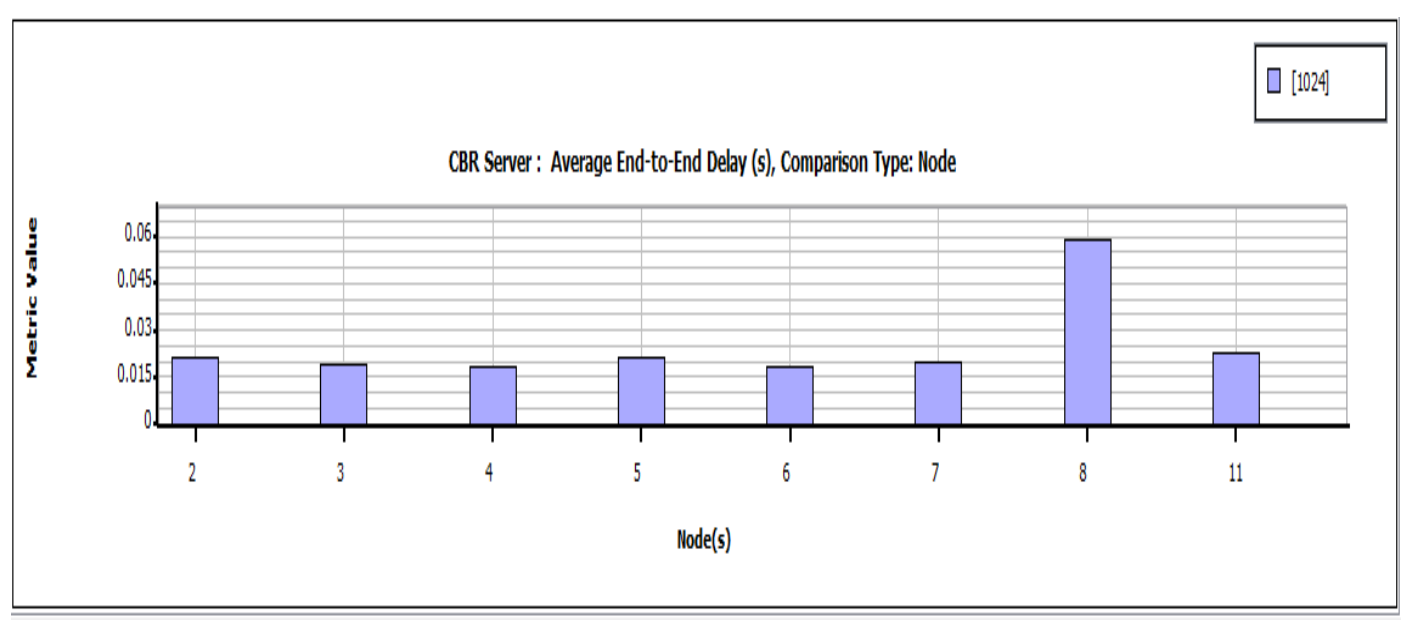

Figure 6. Average End-to-End Delay 
In Figure 6, all sensor nodes are sending packets to base station without delay. Figure 6 show average End-to-End Delay. Average end-to-end delay is time in which data send from sensor node to the base station.

\section{Conclusion}

In this paper, we have proposed a cross layer design approach (IQOS) for improve energy-efficiency and system throughput in wireless sensor network. Proposed IQOS will optimize energy consumption at Physical layer, Data link layer and Network layers of the Wireless sensor network protocol stack (Simplified Protocol Stack of OSI Model). Proposed IQOS allow Communication between layers non-adjacently and each layer has knowledge about other layer like Application layer has knowledge about MAC Layer and MAC Layer has knowledge about Network layer and Network layer has knowledge about Physical and Data link layer. In Proposed cross layer design approach, Scheduling algorithm make schedule to all sensor nodes that are coming from network layer and cross layer allocation scheme allow multiuser transmission in a single communication channel. Proposed IQOS is more energy-efficient approach as compare to existing approach. Proposed IQOS will improve quality of service for wireless sensor network.

\section{Ethics}

This Research paper is original and not published in any conferences or in any journal.

\section{Acknowledgment}

Our special thanks to Prof (Dr.) K.K.Raina, Vice-chancellor, DIT University, Dehradun for his support and guidance.

\section{References}

[1] A. Ahmed Abbasi and M. Younis, "A survey on clustering algorithms for wireless sensor networks", Computer communications, vol. 30, no. 14, (2007), pp. 2826-2841.

[2] J. N. Al-Karaki and A. E. Kamal, "Routing techniques in wireless sensor networks: a survey", Wireless communications, IEEE, vol. 11, no. 6, (2004), pp. 6-28.

[3] A. Sepasi Zahmati, N. M. Moghadam and B. Abolhassani, "Epmplcs: An efficient power management protocol with limited cluster size for wireless sensor networks", Distributed Computing Systems Workshops, ICDCS '07. 27th International Conference on IEEE, (2007).

[4] W. B. Heinemann, A. P. Chandrakasan and H. Balakrishnan, "Application-specific protocol architecture for wireless micro sensor networks", Wireless Communications, IEEE Transactions, vol. 1, no. 4, (2002), pp. 660-670.

[5] W. Rabiner Heinemann, A. Chandrakasan and H. Balakrishnan, "Energy-efficient communication protocol for wireless micro sensor networks", System sciences, 2000, Proceedings of the 33rd annual Hawaii international conference on IEEE, (2000).

[6] A. Sepasi Zahmati, B. Abolhassani, A. Asghar Behesti Shirazi and A. Shojaee Bakhtiari, "An EnergyEfficient protocol with Static clustering for Wireless Sensor Network", Proceedings of world academy of science, Engineering and Technology, ISSN 1307-6884, vol. 22, (2007) July.

[7] S. Ghiasi, "Optimal energy aware clustering in sensor networks", Sensors, vol. 2, no. 7, (2002), pp. 258269.

[8] I. AI-Anbagi, M. Erol-Kantarci and H. T. Mouftah, "A Survey on Cross-Layer Quality of Service Approaches in WSNs for Delay and Reliability-Aware Applications", Proceedings of IEEE, (2014).

[9] Q. Liu, X. Wang and G. B. Giannakis, "A Cross-Layer Scheduling Algorithm with QoS Support in Wireless Networks", Proceedings of IEEE, (2006).

[10] A. K. Demir, H. Engin Demiray and S. Baydere, "QoSMOS: cross-layer QoS architecture for wireless multimedia sensor networks", Springer, Wireless Network, vol. 20, (2014), pp. 655-670.

[11] G. A. Shah, W. Liang and X. Shen, "Cross-Layer Design for QoS Support in Wireless Multimedia Sensor Networks", Proceedings on the IEEE, (2010).

[12] R. Ranjan and S. Varma, "Challenges and Implementation on Cross Layer Design for Wireless Sensor Networks", Springer Business Media New York, (2015).

[13] V. Sentongo and A. Chan, "Optimization of Quality of Service Requirement for Real-Time Applications using Cross Layer Design”, IEEE AFRICON, (2009). 
[14] R. Laufer, T. Salonidis, H. Lundgren and P. Le Guyadec, "Cross-Layer Backpressure Architecture for Wireless Multihop Networks", IEEE/ACM Transactions on Networking, vol. 22, no. 2, (2014) April.

[15] D. Espes, X. Lagrange and L. Suarez, "A cross-layer MAC and routing based on slotted aloha for wireless sensor networks", Springer, Ann.Telecommun, vol. 70, (2015), pp. 159-169.

[16] R. Babaee and N. C. Beaulieu, "Cross-Layer Design for Multihop Wireless Relaying Networks", IEEE Transactions on Wireless Communications, vol. 9, no. 11, (2010) November.

[17] I. F. Akyildiz, M. C. Vuran and Ö. B. Akan, "A cross-layer protocol for wireless sensor networks", Information Sciences and Systems, 2006 40th Annual Conference on IEEE, (2006).

[18] C.-H. Tsai, "Cross-layer, energy-efficient design for supporting continuous queries in wireless sensor networks: a quorum-based approach", Wireless personal communications, vol. 51, no. 3, (2009), pp. 411-426.

[19] R. Ranjan and S. Varma, "Challenges and Implementation on Cross Layer Design for Wireless Sensor Networks”, Wireless Personal Communications, Springer Business Media New York, (2015), pp. 1-24.

\section{Authors}

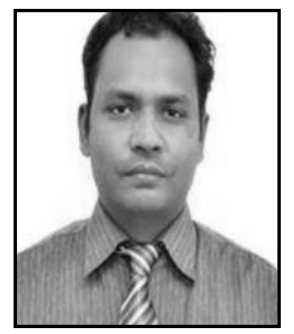

Rakesh Kumar Saini, received the MCA degree from UPTU, Lucknow, India in 2005 and M.Tech (Computer Science and Engineering) degree from UTU, Dehradun, India in 2012 and Pursuing PhD from DIT University, Dehradun, India in 2014.He is having 12 Years of teaching experience. He is author of around 10 books. His research interests include Wireless Sensor Network, Energy-Efficiency in Wireless Sensor Network.

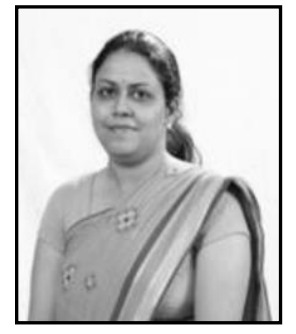

Dr. Ritika, received the Ph.D. degree in Computer Science from GKU, University, Haridwar in the year 2010, M.Tech degree in Computer Science and Engineering from UTU, Dehradun and MCA from GKU Dehradun. She is life time membership of ISCA, CSI, IEEE, IAENG, ISCA and IETE. She was Chairman of Computer Society of India Dehradun Chapter in the year 2013. She specializes in core areas of computer science and holds experience of more than 14 years. 